\title{
Unsteady Mixing of Binary Liquid Mixtures with Composition-Dependent Viscosity
}

\author{
Chiara Galletti ${ }^{1}$, Elisabetta Brunazzi, Roberto Mauri \\ Department of Civil and Industrial Engineering, Laboratory of Multiphase Reactive Flows, \\ Università di Pisa, Largo L. Lazzarino 2, I-56122 Pisa, Italy
}

\begin{abstract}
The laminar mixing of two miscible liquids in a $\mathrm{T}$ junction is investigated through Direct Numerical Simulations. In particular the liquids have the same density and the same viscosity, but the viscosity of the mixture strongly depends on composition and can be up to three times larger or smaller than that of the pure liquids; this is aimed at mimicking the behavior of many aqueous and organic mixtures of interest in the pharmaceuticals and fine chemistry industry. The mixing process is found to be largely affected by the dependence of viscosity on composition. When the viscosity of the mixture is larger than that of the pure components, a viscous layer forms at the confluence of the inlet flows, which tends to keep the two streams separated, resulting in a shift of the engulfment regime towards larger Reynolds numbers. On the contrary, when the viscosity of the mixture is lower than that of the pure components, an acceleration of the uid at the center of the mixing channel takes place, leading to an enhancement of the degree of mixing. At larger Reynolds number, unsteady, time periodic flow regimes, able to strongly increase the degree of mixing, are established for all types of mixtures. Although fluid oscillations are all characterized by typical Strouhal numbers lying in the range $0.1-0.3$, the onset of the unsteady regimes, together with the corresponding vortical structures, strongly depends on the fluid rheology.
\end{abstract}

\footnotetext{
${ }^{1}$ Author to whom correspondence should be addressed. Electronic mail: chiara.galletti@unipi.it.
}

Preprint submitted to Chemical Engineering Science

December 21, 2016 
Keywords: Micromixing; Multiphase flow; Laminar flow; Computational Fluid Dynamics; T-junction.

\section{Introduction}

A fast and efficient mixing at the microscale is required in many processes ranging from miniature fuel cells to molecular diagnostics. Among passive micro-mixers, where mixing is promoted without the help of any external power source (Kumar et al., 2011), one of the simplest designs is a T shape, in which the inlets join the main channel with T-shaped branches. This type of mixer is also suitable for carrying out fundamental studies, as it is often the junction element in more complex micro-systems. The efficiency of T-shaped micro-mixers for liquid mixing has been largely investigated in the literature and different flow regimes have been identified, both numerically (Bothe et al., 2006; Dreher et al., 2009; Fani et al., 2014; Andreussi et al., 2015) and experimentally, using water as working fluid (see $\mu$-LIF and $\mu$-PIV experiments by Hoffmann et al., 2006, and the flow visualizations by Engler et al., 2004). In particular, it was shown that the inlet streams remain separated up to a critical Reynolds number, $R e=140$, corresponding to the transition from a vortex flow regime, with a double mirror symmetry, to an engulfment flow regime, with a point central symmetry, leading to a large increase of the degree of mixing. Then, a few investigations have indicated that by further increasing $R e$, the flow becomes first unsteady and time-periodic at $R e=240$, and eventually chaotic at $R e=500$ (Dreher et al., 2009; Thomas et al., 2010; Thomas and Ameel, 2010; Kockmann and Roberge, 2011; Minakov et al., 2012, 2013). These results were explained by Fani et al. (2014) and extended by Andreussi et al. (2015) who investigated the effect of geometry on the flow regimes. In particular, in agreement with Thomas et al. (2010) and Thomas and Ameel (2010), they observed an unexpected transition from an unsteady asymmetric regime, i.e. presenting vortical structures with two main legs in the mixing channel, to an unsteady symmetric regime, i.e. characterized by four main legs with a double-mirror symmetry, 
which may dampen the mixing process.

The practical use of micro-devices involves mixing of two different fluids, often organic ones, as it is the case in pharmaceutical and fine chemistry industry. Hence the validity of the aforementioned results, obtained by feeding the same fluid, i.e. water, from both inlets, should be checked when using practical fluids and mixtures, that often show an non-ideal behavior, i.e. the viscosity is not a linear function of the composition. In a recent work (Galletti et al., 2015), we studied the engulfment process in a T-shaped micro-device for a model binary mixture, composed of two fluids having the same density and the same viscosity, yet presenting a strong fluidity of mixing effect, i.e., the viscosity of the mixture is a function of its composition. In the case of a positive fluidity of mixing, when the fluid mixture has a smaller viscosity than that of its pure components, the onset of the engulfment regime is accompanied by a sharp increase of the degree of mixing, with the critical Re decreasing as the fluidity of mixing increases. The contrary occurs when the fluid mixture has a larger viscosity than that of its pure components. This result is in agreement with previous findings by Orsi et al. (2013a), who compared the case where the two inlet fluids are both water with that where one inlet fluid is water and the other is ethanol, finding that the vortex-engulfment flow transition occurs at larger Re numbers for the water-ethanol case than for water-water case (i.e., $R e=230$ vs. $R e=140$ ). The reason of this result is that a water-ethanol mixture has a viscosity that is up to three times larger than that of water, so that at the confluence of the T-mixer, the two streams are kept separated from each other by a viscous interfacial layer that hampers vortex formation and retards mixing (Orsi et al., 2013b). However, it was also observed that at low Re the presence of viscosity gradients ensures some degree of mixing, albeit small.

In the present work, we want to corroborate previous findings and better investigate the dependence of the mixing process on the shape of the viscositycomposition diagram $\mu=\mu(\phi)$. In addition, we want to investigate how the mixing process takes place at larger $R e$, when the flow regime becomes first time periodic and then chaotic. Hence, we intend to characterize the transitions 
between different types of steady and unsteady regimes and how they depend on the mixture rheology; this will provide a large database, encompassing all cases that might be of interest in microfluidics.

\section{Problem description}

\subsection{Constitutive relations}

In order to investigate the effect of a composition-dependent viscosity on mixing, we assume that the two fluids composing the mixture have the same density, $\rho$, and the same viscosity, $\mu_{0}$, and that, upon mixing, density remains constant, while the mixture is a Newtonian fluid, with a composition-dependent viscosity. The following types of constitutive relations are considered:

B base case, for which $\mu(\phi)=\mu_{0}$;

N1 negative fluidity of mixing, with a symmetric quadratic composition diagram,

$$
\mu(\phi)=\mu_{0}\left[1+K_{1} \phi(1-\phi)\right] ; \quad K_{1}=(\alpha-1) / \beta^{2} .
$$

N2 negative fluidity of mixing, with a symmetric and sharp composition diagram,

$$
\mu(\phi)=\mu_{0}\left[1+K_{2} \phi^{3}(1-\phi)^{3}\right] ; \quad K_{2}=\beta^{-7}
$$

S sinusoidal viscosity composition diagram,

$$
\mu(\phi)=\mu_{0}\left[1+K_{3} \sin (2 \pi \phi)\right] ; \quad K_{3}=\frac{1}{2} .
$$

P1 positive fluidity of mixing, with a symmetric quadratic composition diagram,

$$
\mu(\phi)=\mu_{0}\left[1-K_{4} \phi(1-\phi)\right] ; \quad K_{4}=(\alpha-1) /\left(\alpha \beta^{2}\right) .
$$

In the N1- and N2-type mixtures, viscosity reaches its maximum value, $\mu_{\text {max }}=\alpha \mu_{\text {min }}=\alpha \mu_{0}=3 \mu_{0}$, at $\phi=\beta=\frac{1}{2}$, that is for a $50 \%-50 \%$ composition; in the P1-type mixture, viscosity presents a minimum value, $\mu_{\min }=\mu_{0} / \alpha$, at 
$\phi=\beta=\frac{1}{2}$, that is for a $50 \%-50 \%$ mixture; in the S-type mixture, viscosity has a maximum, $\mu_{\max }=\frac{3}{2} \mu_{0}$, at $\phi=\frac{1}{4}$ and a minimum, $\mu_{\min }=\frac{1}{2} \mu_{0}$, at $\phi=\frac{3}{4}$. The viscosity-composition diagram is reported in Fig. 1 for each mixture type. Note that, in all cases, $\mu_{\max } / \mu_{\min }=3$; this choice was made to emulate the behavior of one of the most common mixtures, namely water-ethanol, whose maximum viscosity is almost three times that of pure water (Simmonds, 1919).

[Figure 1 about here.]

Examples of N-type mixtures are many aqueous solutions of organic solvents, such as ethanol, acetone, methanol, propanol and acetic acid (Laliberté, 2007); ethanol / methylacetate and ethanol / benzene are examples of P-type mixtures, while benzene / methanol and toluene / methanol are examples of S-type mixtures (see Fig. 2 of Gonzalez et al., 2007, and references therein; Figs. 1-4 of Lei and Hou, 1999).

The main idea behind these simulations is to investigate the dependence of the degree of mixing on the viscosity gradients. In fact, during the mixing process of a fluid mixture, convective flows are induced by viscosity gradients, due to the non-homogeneous distributions of composition and temperature. Accordingly we expect that, for example, comparing the $\mathrm{N} 1$ with the $\mathrm{N} 2$ case, as in the latter the viscosity gradient reaches a larger value than in the former case, the convective flux will be larger, therefore enhancing the mixing process.

\subsection{T-mixer geometry}

[Figure 2 about here.]

The geometry of the problem consists of the T-shaped micro-mixer illustrated in Fig. 2, where the coordinate $y$ denotes the distance from the confluence of the inlet streams. Here, the inlet channels have a square cross section, with $W_{i}=H$, while the mixing channel presents a $2: 1$ aspect ratio, i.e. $W=2 H$, so that the hydraulic diameter is $d=4 H / 3$. The length of the inlet channel, $L_{i}=2.25 d$, was chosen to include the whole confluence region, while the length 
of the mixing channel, $L=15 d$, is long enough to completely describe the complex vortical structures, ensuring that the channel length does not influence the simulation results. Taking $H=100 \mu m$, the geometric setup is the same as used by many researchers (see, for example, Bothe et al., 2006, and Hoffmann et al., 2006). Further details about the geometric setup can be found in Galletti et al. (2012, 2015).

\subsection{Governing equations}

The motion of an incompressible fluid mixture is described by the nondimensional Navier-Stokes equations,

$$
R e\left(\frac{\partial \mathbf{v}}{\partial t}+\mathbf{v} \cdot \nabla \mathbf{v}\right)=\nabla \cdot \mathbf{T} ; \quad \nabla \cdot \mathbf{v}=0
$$

Here dimensions, velocities and time are scaled in terms of the mixing channel hydraulic diameter $d$, the average mean velocity $U$, and the convective time $d / U$, so that the Reynolds number is defined as $R e=\rho U d / \mu_{0}$, where $\rho$ is the constant, uniform density. In addition, $\mathbf{T}$ is the stress tensor which, assuming the mixture to be a Newtonian fluid with a composition-dependent viscosity, satisfies the constitutive equation,

$$
\mathbf{T}(\phi)=-P \mathbf{I}+\tilde{\mu}(\phi)\left(\nabla \mathbf{v}+\nabla \mathbf{v}^{T}\right),
$$

where $P$ is the modified non-dimensional pressure, $\phi$ the mass (and volume)

fraction of, say, component $A$ of the binary mixture, the $T$ superscript indicates the transpose of a tensor, while $\tilde{\mu}=\mu / \mu_{0}$ is the non-dimensional viscosity.

The Navier-Stokes equations are coupled with a conservation equation of the chemical species,

$$
P e\left(\frac{\partial \phi}{\partial t}+\mathbf{v} \cdot \nabla \phi\right)=D \nabla^{2} \phi
$$

where $P e=U d / D$ is the Peclet number, with $D$ denoting the molecular diffusivity. If the two fluids are identical, we can imagine adding a very small amount of contaminant, i.e. a dye, to one of the fluids (which therefore continue to have the same physical properties) and therefore, in this case, $\phi$ indicates the (normalized) dye mass fraction. Finally, we consider that in liquid mixtures 
the Schmidt number is very large, i.e. $S c=\mu_{0} /(\rho D) \gg 1$, so that $P e \gg R e$. Equations (5)-(7) are subjected to boundary conditions, consisting of no-slip velocity, no-mass-flux at the channel walls, and a constant ambient pressure at the exit, while at the entrance a flow profile corresponding to fully developed flow conditions in a square conduit (Happel and Brenner, 1965) is imposed through a bespoke subroutine as also described in Galletti et al. (2012). The two fluids are fed with the same flow rate.

\subsection{Vorticity structures and degree of mixing}

In the following, the morphology of the flow field will be visualized using the $\lambda_{2}$-criterion (Jeong and Hussain, 1995), according to which a vortex is defined as a connected fluid region where the second largest eigenvalue of the symmetric tensor $\mathbf{L}=\mathbf{S} \cdot \mathbf{S}+\mathbf{A} \cdot \mathbf{A}$ is negative, i.e. $\lambda_{2}<0$. Here, $\mathbf{S}$ and $\mathbf{A}$ indicate the symmetric and anti-symmetric part of the velocity gradient, that are the strain rate and vorticity tensors, respectively, i.e., $\nabla \mathbf{v}=\mathbf{S}+\mathbf{A}$, and therefore $\lambda_{2}$ is the Galileian invariant second solution of the cubic characteristic equation of $\mathbf{L}$. At the end, since we must express quantitatively the efficiency of the mixing process, we define the standard deviation of the volumetric flow on the cross section at the exit of the outlet channel, $\sigma_{b}$, as:

$$
\sigma_{b}^{2}(y)=\frac{1}{N \bar{u}} \sum_{i}\left(\phi_{i}-\bar{\phi}_{b}\right)^{2} u_{i} ; \quad \overline{\phi_{b}}=\frac{\sum_{i} \phi_{i} u_{i}}{N \bar{u}}
$$

where $\bar{\phi}_{b}$ is the bulk, or cup mixing average, mass fraction (Middleman, 1998), $u_{i}$ is the axial velocity of the mixture at point $i$ within the cross section, while $\bar{u}=\sum_{i} u_{i} / N$ is its mean value, i.e., the ratio between the volumetric flux and the cross section area. Finally, as in Orsi et al. (2013a), we define the degree of mixing $\delta_{m}$ on the cross section at the exit of the outlet channel as:

$$
\delta_{m}=1-\frac{\sigma_{b}}{\sigma_{\max }} ; \quad \sigma_{\max }=\sqrt{\bar{\phi}_{b}\left(1-\bar{\phi}_{b}\right)},
$$

where $\sigma_{\max }$ is the maximum value of the standard deviation $\sigma$, that is achieved when the two inlet streams remain completely segregated. 


\section{Numerical model}

\subsection{Setup}

Simulations were performed using the commercial software Fluent v. 15 by ANSYS Inc.. A grid independence study on the velocity field was performed at the highest $R e$ that was used in our simulations, leading to choose cubic elements with $H / 20$ edge, corresponding to $20 \times 40$ cells in each cross section of the mixing channel, in agreement with the work of Hussong et al. (2009). A stationary solver with a second order discretization scheme was used for simulating the steady regimes (i.e., in the vortex and engulfment regimes). The SIMPLE algorithm was employed to handle the pressure-velocity coupling. At convergence the normalized residuals for velocities were stationary with iterations and most of the times fell below $1 \times 10^{-10}$. Such small residuals were set in order to ensure converged solutions near the engulfment, where larger values of residuals would led to the prediction of unstable mixing pattern (Galletti et al., 2012). The steadiness of the solution was also checked by monitoring velocity and concentration values in some locations in the mixing channel at different iterations. In unsteady conditions (i.e., in the periodic and chaotic regimes), a second order implicit solver was used, with a maximum Courant number of about 5 . The stability of statistical values was checked; moreover simulations in periodic regime were run to cover at least 20 cycles to allow frequency analysis of time series.

\subsection{Validation}

As mentioned above, the concentration field was discretized using the same mesh size as for the velocity field. An extended discussion to justify this assumption was presented in Galletti et al. (2015) considering that, in liquid mixtures, the Schmidt numbers is very large, i.e. $S c \gg 1$. In fact, as shown in Orsi et al. (2013a) through a regular perturbation analysis, at leading order the diffusion flux in Eq. (7) can be neglected, provided that it is not identically perpendicular to the convective flux. Accordingly, the B-type mixture constitutes the most critical case, as there is no transversal fluid convection induced by 
viscosity gradients, that otherwise could prevent the formation of destabilizing sharp concentration gradients.

The results for the B-type mixture are in agreement with experimental and numerical investigations, as described in Section 3.1 of our previous work (Galletti et al., 2015), so that the numerical scheme can be considered reliable.

In order to validate our results in case of composition-dependent viscosity, some of the simulations were performed with a different code, i.e. Nek5000 (https://nek5000.mcs.anl.gov). Nek5000 is an open-source massive parallel code based on an high order spectral element method (Fisher, 1997); therefore it differs largely from Fluent, that is a finite volume solver. In Nek5000 the spatial discretization of the domain consists of a large number of hexahedral elements; the unknown variables are spanned by $N$ th-order Lagrange polynomial interpolants, based on tensor-product arrays of Gauss-Lobatto-Legendre quadrature points in each spectral element, where typically $N>6$. A third order backward differential formula (BDF3) is employed for the time derivatives. The diffusive terms are treated implicitly, while a third order explicit extrapolation (EXT3) is considered for the convective terms. This explicit extrapolation for the convective terms in the BDF3-EXT3 scheme imposes a time-step restriction; in particular, the time step here is chosen in order to satisfy the condition of Courant number being equal to 0.5 . The computational domain consists in a structured multi-block grid constituted by 16000 spectral elements. The polynomial order $N$ for the velocity is fixed to 8 , while an order of 6 is imposed for the pressure, thus using the common $P_{N} / P_{N-2}$ scheme, for a total of about $6 \cdot 10^{6}$ degrees of freedom (dofs) for each velocity component and $3.5 \cdot 10^{6}$ dofs for the pressure. A comparison of vortical structures in the mixing channel, as identified through the $\lambda_{2}$ - criterion, obtained using the two codes (i.e, Fluent and Nek5000) is reported in Fig. 3 for the N1-type mixture at $R e=400$. The vortical structures obtained with Fluent have been colored in red in order to facilitate the comparison with the Nek5000 results, whereas they will be represented in gray in the following graphs. Here the morphology of the flow structure will not be discussed, however it is evident an excellent agreement between the 
flow structures obtained with two completely different numerics. Please note that the two simulations agree even in predicting the presence of small spots on the upper side of the main vortical legs.

[Figure 3 about here.]

\section{Results}

\subsection{Steady regimes}

As we have previously mentioned, the results of the B-type mixture are in perfect agreement with previous investigations, thus validating our numerical scheme. Therefore, this will be considered as our base case, against which the behavior of the other mixture types will be compared.

In Fig. 4 the concentration distributions in the cross-section of the mixing channel located just downstream of the confluence region, at $y=1.5 d$, are shown for different Reynolds number, in all cases under investigation.

[Figure 4 about here.]

In the B-type fluid, corresponding to a mixture of two liquids having the same viscosity (and density), the results clearly indicate a segregated flow in the vortex regime at $R e=125$ (Fig. 4a) and a symmetry breaking at $R e=150$, 160 and 175 (Figs. 4b-d), which is typical of the engulfment regime.

In the N1-type mixture, characterized by a negative fluidity of mixing with a quadratic dependence of viscosity upon composition, some mixing is present even at flow rates as low as $R e=125$ (Fig. 4e), due to the transverse flow induced by viscosity gradients, although at larger Reynolds numbers the flow pattern appears less swirled than the ones for the B-type mixture (see Fig. 4g at $R e=160)$.

In the N2-type mixture, with negative fluidity of mixing and a viscosity dependence on composition described by a 6 -th order polynomials, instead, we observe a different behavior than in the N1-type mixture. In fact, the flow is well segregated at $R e=150$ (Fig. 4j), with the onset of the engulfment regime 
occurring at $R e=160$ (Fig. 4k), i.e. at larger $R e$ than in N1-type mixture. This indicates that the presence of a well defined region with a high viscosity hampers mixing and delays the onset of the engulfment regime. The interface between two liquids in Fig.4i,j appears sharper than that of the B-type (Fig.4a), as a refinement of the grid based on concentration gradients was necessary for the N2-type mixture in vortex regime, to avoid convergence problems, that were due to the steep dependence of viscosity on concentration approaching the $50 \%$ - $50 \%$ composition. As a consequence, these simulations are also affected by lower numerical diffusion.

The S-type mixture shows some mixing at $R e=125$ (Fig. 4m), as the flow field is not as segregated as in the B-type mixture. Moreover, the pattern exhibits just a single (mirror) symmetry that evolves to become a central point symmetry at $R e=175$ (Fig. 4p).

Finally, in the P1-type mixture with positive fluidity of mixing and a quadratic dependence of viscosity upon composition, we observe high degrees of mixing even at $R e=125$ (Fig. 4q). The P1-type mixture at $R e=175$ is not shown here as for such Reynolds number the flow is unsteady.

These results are confirmed and clarified by carefully examining the vortical structures and the y-vorticity at the confluence represented in Fig. 5, as identified through the $\lambda_{2}$-criterion described in Section 3.2.

[Figure 5 about here.]

First, in Figs. 5a-c, the evolution of the vortical structures in the B-type mixture are shown, as we pass from the vortex to the engulfment regime. In the vortex regime (Fig. 5a), the vortical structures are characterized by four legs of the same strength, corresponding to a double pair of counter-rotating vortices. Then, as Re is increased, a tilting of the vortical structures is observed at the confluence of the inlet channels, leading to two stronger legs that extend into the mixing channel, together with other two weaker legs (see Fig. 5b at $R e=175$ ). Such flow structures are very similar to the ones observed by Fani et al. (2013), who used a T-mixer with a different aspect ratio. Finally, further increasing the 
flow rate up to $R e=225$, we observe a complex vortical structure, composed of a double pairs of vortices that swirl and recombine, generating a pair of stronger legs. Moreover, there is an additional pair of vortical structures near the center of the mixing channel, leading to a pair of weaker legs, thus preceding the onset of the unsteady regime that occurs at $R e=240$.

The behavior of the N1-type mixture is depicted in Figs. 5d-f. At Re $=150$ the perfect double mirror symmetry of the vortex regime is already lost. This can be observed from the $\mathrm{y}$-vorticity at $y=3 d$. Then, increasing the flow rate to $R e=175$, a tilting of the structures is observed, although the strength of the four legs is approximately the same. Finally, with a further significant increase of the flow rate, we observe two dominant legs and two weaker legs near the center of the mixing channel (see Fig. $5 \mathrm{f}$ at $R e=400$ ).

The N2-type mixture (Figs. 5g-i) presents vortical structures that are much different from those of the N1-type mixture, thus confirming the results of Fig. 4. At $R e=150$ we see a double mirror symmetry that resembles the one of the vortex regime in the B-type mixture at $R e=125$. Then, increasing $R e$, a symmetry breaking takes place, leading to the appearance of two main legs; indeed the vortical structures of Fig. 5h are very similar to those of Fig. 5b for the B-type mixture. Finally, further increasing $R e$, the structures become more complex.

The S-type mixture (Figs. 5j-1) shows just a single mirror symmetry in the vortex regime (see Fig. $5 \mathrm{j}$ at $R e=125$ ) because the viscosity-composition diagram is not symmetric. Indeed the two stronger legs of the engulfment regime have different magnitude (see Fig. $5 \mathrm{k}$ at $R e=167$ ). However, again we notice that before the onset of the unsteady regimes, the structures become more complex, as in the confluence region we observe a double-pair of swirling vortices that recombine, forming two stronger legs, together with a pair of weaker vortices represented by the smaller legs.

Finally, P1-type mixture (Figs. 5m-o) shows very complex patterns even at flow rates as small as at $R e=125$. 


\subsection{Unsteady regimes}

The unsteady regime was observed for all types of mixtures, although its onset is strongly affected by the viscosity-concentration diagram. The values of Re corresponding to the onset of unsteady and chaotic regimes are reported in Table 1 for each type of mixture. For the B-type mixture, many investigators indicated that the unsteady regime occurs at $R e=240$ (Bothe et al., 2006; Dreher et al., 2009; Andreussi et al., 2015) and the chaotic regime at $R e=515$ (Dreher et al., 2009; Andreussi et al, 2015). It can be observed that the N1and N2-type mixtures, with negative fluidity of mixing, are both characterized by a significant increase of the flow rates at which the unsteady regime starts to occur, compared to the B-type mixture. For example, the onset of the unsteady regime for the N1-type mixture occurs at $R e=550$, hence at a much larger flow rate than for the B-type mixture (i.e., at $R e=240$ ). Note that in this case the range of $R e$ at which the flow regime is periodic is rather narrow, since at $R e=650$ the flow becomes chaotic. Similar considerations hold for the N2type mixture as well, even though the onset of the unsteady regime in this case is anticipated to $R e=450$, compared to $R e=550$ for the N1-type mixture. Hence, we may say that the presence of mild viscosity gradients retards the onset of an unsteady flow regime.

The P1-type mixture shows an unsteady regime at flow rates as low as $R e=$ 175 , whereas the flow becomes chaotic at $R e=350$. The presence of periodic motions was observed also in case of non symmetric viscosity-concentration diagram, as for the S-type mixture. In this case, the flow becomes periodic at $R e=300$ and subsequently chaotic at $R e=450$.

[Table 1 about here.]

Fig. 6 shows some snapshots, illustrating the vortical structures in the mixing channel at different times for N1- (on the left) and N2-type (on the right) mixtures. In both cases it can be observed the presence of two main legs, indicating a twisted asymmetric pattern. Although the two sets of figures refer to different flow rates, i.e., $R e=600$ and $R e=500$ for N1- and N2-type mixtures, 
respectively, the kind of oscillations appears similar. The only noticeable difference is that the legs in the N1-type mixture appears less swirled than in the N2-type mixture (compare for instance, Fig. 6g and Fig. 6h), probably due to the presence of a well defined viscous layer separating the incoming flows in the latter mixture. However this statement should be verified further.

[Figure 6 about here.]

Similarly, Fig. 7 shows some snapshots of the vortical structures for the P1type mixture at $R e=250$ (on the left) and $R e=300$ (on the right). It is evident that the flow morphology is much different from that of the N-type mixtures, presenting stronger inner legs, i.e., higher vorticity, at the center of the mixing channel, where viscosity is low. In the N-type mixtures, instead, the external legs are always the dominating ones, with weaker inner legs, due to the high viscosity region at the center of the channel. Thus, we may conclude that the low viscosity at the center of the channel characterizing P1-type mixtures induces an acceleration of the flow field, with consequent stronger vortical structures in that region. In fact, increasing $R e$, we see that those inner legs may prevail over the external ones, leading to the patterns shown in Fig. 7 .

[Figure 7 about here.]

The characteristic frequency $f$ of the periodic motions was evaluated by analyzing the time-series of the $y$ velocity component in the mixing channel axis near the confluence at $y=0$ and $y=1.5 d$, using Matlab® routines based on Fast Fourier Transforms. Time series of the $y$ component of the instantaneous velocity, $v_{y}$, at $y=0$ are shown for the N1-type mixture and for different $R e$ in Fig. 8. The $R e=550$ and $R e=600$ time series show a perfect periodicity that it is slightly lost when increasing $R e$ and thus moving towards the chaotic regime.

[Figure 8 about here.] 
Fig. 9 reports the Strouhal number, based on the mixing channel hydraulic diameter and the mean velocity,

$$
S t=\frac{f d}{U}
$$

For the B-type mixture, increasing Re first results in an increase of the Strouhal number from $S t=0.15$ to $S t=0.28$, and subsequently in a gradual decrease, reaching $S t=0.26$ at $R e=450$. Such mild reduction is associated to the previously mentioned morphology change, from an asymmetric to a symmetric periodic regime, the latter being characterized by a double pair of counter-rotating vortices (see Andreussi et al., 2015).

[Figure 9 about here.]

All investigated mixtures provide a dependence of $S t$ on $R e$ similar to the B-type mixture in the range $S t=0.15-0.3$, with the exception of the P1type mixture. In fact, this mixture shows, surprisingly, after an initial gradual increase of $S t$, reaching $S t=0.27$ at $R e=250$, a sharp decrease to $S t=0.11$ at $R e=300$. Similar reduction on the $S t$ number has been observed by Thomas and Ameel (2010), Thomas et al. (2010) and Andreussi et al. (2015) and was explained by a transition from an unsteady asymmetric regime, with two vortical legs in the mixing channel, to an unsteady symmetric regime with four main legs. However, the vortical structures of the P1-type mixture (see Fig. 7) indicate an asymmetric flow for both $R e=250$ and $R e=300$. At such $R e$ very strong vortical structures are observed near the center of the mixing channel, manifesting a fluid acceleration that may hamper transversal oscillations, thus reducing $S t$. This at first unexpected behavior will be further investigated in future works.

\subsection{Degree of mixing}

Fig. 10 reports the degree of mixing evaluated at the outlet of the mixing channel as a function of $R e$ for all types of mixtures. The solid and dashed lines refer to the steady and the unsteady flow regimes, respectively. Note that the 
degree of mixing in the unsteady regime is determined within an uncertainty, represented by error bars, due to the fluctuations of the flow field. Such error bars were determined by analyzing the velocity and concentration fields at 18 - 20 different times uniformly distributed within a flow cycle (whose extent was determined from the frequency analysis). Hence the degree of mixing at the outlet section was evaluated for each acquisition, to estimate its average, minimum and maximum value. The error bars represent the difference between the maximum and minimum degree of mixing.

[Figure 10 about here.]

For the B-type mixture at $R e=138$ a well-known sudden increase of the degree of mixing is observed, due to the onset of the engulfment regime.

The N1-type mixture shows instead a gradual increase of the degree of mixing. At $R e=125$ there is some degree of mixing with respect to the B-type mixture, because of the transversal mass flux induced by viscosity gradients that promotes mixing. Then, at larger $R e$, mixing increases gradually, but it always remains lower than in the B-type mixture, because of the presence of a separating viscous layer between the two converging fluids, that hampers the development of a strong engulfment flow. For instance, the degree of mixing at $R e=200$ is $\delta_{m}=45 \%$ for the B-type mixture whereas it is just $\delta_{m}=28 \%$ for the N1-type mixture. The gradual increase of mixing leads to $\delta_{m}=50 \%$ at $R e=500$. Further increasing $R e$, then, the flow becomes unsteady at $R e=550$ with a degree of mixing larger than $70 \%$.

The N2-type mixture, although it is characterized by the same negative fluidity of mixing as the N1-type mixture, shows a much different behavior, thus highlighting that the shape of the viscosity-concentration diagram is also important. In this case, mixing is nearly zero at low $R e$, and then increases suddenly at the engulfment. Then, unsteady motions lead to a increase of the degree of mixing from $50 \%$ to approximately $80 \%$.

Contrary to the behavior of N-type, the P1-type mixture shows a very sharp engulfment point at $R e=118$; in addition, the onset of the unsteady flow 
regimes (both time periodic and chaotic) are also anticipated compared to the B-case.

\section{Conclusions and discussion}

The behavior of different binary mixtures in a T-shaped micro-device was investigated numerically in order to understand how the fluidity of mixing, i.e. the dependence of viscosity $\mu$ upon composition $\phi$, affects the mixing process. Hence, the mixtures consist of two miscible fluids having the same density, $\rho_{0}$, and the same viscosity, $\mu_{0}$, but presenting a strong fluidity of mixing effect. Specifically, we took into account the following cases: a) $\mu=\mu_{0}$ (this is the base case, indicated as the B-type mixture); b) $\mu$ is larger than $\mu_{0}$, reaching a maximum value, $\mu_{\max }=3 \mu_{0}$ at $\phi=\frac{1}{2}$ more or less gradually (N1- and N2-type mixtures); c) $\mu$ is smaller than $\mu_{0}$, reaching a minimum value, $\mu_{\min }=\frac{1}{3} \mu_{0}$,

at $\phi=\frac{1}{2}$ (P1-type mixture); d) $\mu$ has a sinusoidal dependence on $\phi$ (S-type mixture).

In the base case, the flow field undergoes a sudden transition from a mirrorsymmetric vortex regime to a point-symmetric engulfment regime at $R e=138$, with a sharp increase of the degree of mixing. Similar behavior is observed with P1-type mixtures, where the onset of the engulfment regime is sharp and occurs at smaller Reynolds number, i.e. $R e=118$. With N1- and N2-type mixtures, such transition occurs gradually and the degree of mixing in the engulfment regime is smaller that the one observed for the base case, due to the formation of a viscous layer at the confluence of the two inlet streams that hampers any further transverse convection. Differences were noticed between N1- and N2type mixtures, indicating that the viscosity gradients affects the mixing process. With S-type mixtures, the transition occurs suddenly, but at larger $R e=160$. Moreover the asymmetry of the mixture rheology prevents the flow field from assuming any double-mirror symmetric morphology, even in the vortex regime.

Further increasing the flow rate well above the onset of the engulfment regime, the flow for all mixture types becomes unsteady, with clear time periodic behaviors. This unsteady regime takes place for all mixtures; for instance, 
its onset occurs at $R e=240$ for the base case, but only at $R e=550$ for the N1-type mixture, thus showing that the range of $R e$ for which periodic oscillations are present strongly depends on the mixture type. In fact, despite the Strouhal number in all cases lays in a narrow range $S t=0.1-0.3$, the vortical structures appear to be quite different. In particular, for the P1-type mixture, the low viscosity region at the confluence promotes a strong fluid acceleration al the center of the mixing channel, thus enhancing the strength of the central vortical structures, whereas in N-type mixtures the fluid flow at the center is slower, thus determining stronger lateral vortices.

Similar remarks can be made concerning the onset of the chaotic regime, occurring at larger $R e$, which is due to the interplay between lateral convection and longitudinal flows; the former is induced by viscosity gradients and, at the same time, it is hampered by the viscous layer separating the two inlet

flows, while the latters are either accelerated or retarded, depending whether the mixtures present positive or negative fluidity of mixing, respectively.

\section{Acknowledgment}

This work was supported by the University of Pisa through the "Progetti di Ricerca di Ateneo 2015" funding program. We also would like to thank Dr. Lorenzo Siconolfi for helping us to run the Nek5000 simulations. 


\section{References}

- Andreussi T., Galletti C., Mauri R., Camarri S., Salvetti M.V., 2015, Flow regimes in T-shaped micro-mixers, Comput. Chem. Eng. 76, 150-159.

- Bothe, D., Stemich, C., Warnecke, H.J., 2006. Fluid mixing in a T-shaped micro-mixer. Chem. Eng. Sci. 61, 2950-2958.

- Bothe, D., Lojewski, A., Warnecke, H.J., 2011. Fully resolved numerical simulation of reactive mixing in a T-shaped micromixer using parabolized species equations. Chem. Eng. Sci. 66, 6424-6440.

- Dizechi, M., Marschall, E., 1982. Viscosity of some binary and ternary liquid mixtures, J. Chem. Eng. Data 27, 358-363.

- Dreher, S., Kockmann, N., Woias, P. 2009. Characterization of Laminar Transient Flow Regimes and Mixing in T-shaped Micromixers. Heat Trans. Eng. 30, 91-100.

- Engler, M., Kockmann, N., Kiefer, T., Woias, P., 2004. Numerical and experimental investigations on liquid mixing in static micromixers. Chem. Eng. J. 101, 315-322.

- Fani, A., Camarri, S., Salvetti, M.V., 2013. Investigation of the steady engulfment regime in a three-dimensional T-mixer. Phys. Fluids 25, 064102.

- Fani, A., Camarri, S., Salvetti, M.V., 2014. Unsteady asymmetric engulfment regime in a T-mixer. Phys. Fluids 26, 074101.

- Fisher, P.F., 1997. An overlapping Schwarz method for spectral element solution of the incompressible Navier-Stokes equations. J. Comput. Phys. $133,84101$.

- Galletti, C., Roudgar, M., Brunazzi, E., Mauri, R., 2012. Effect of inlet conditions on the engulfment pattern in a T-shaped micro-mixer. Chem. Eng. J. 185-186, 300-313. 
- Galletti C, Arcolini G., Brunazzi E, Mauri R., 2015. Mixing of binary fluids with composition-dependent viscosity in a T-shaped micro-device, Chem. Eng. Sci. 123, 300-310.

- González, B., Calvar, N., Gómez, E., Domínguez, A., 2007. Density, dynamic viscosity, and derived properties of binary mixtures of methanol or ethanol with water, ethyl acetate, and methyl acetate at $\mathrm{T}=293.15$, 298.15, and 303.15 K, J. Chem. Thermodyn. 39, 1578 - 1588.

- Happel, J., Brenner, H., 1965. Low Reynolds Number Hydrodynamics, Prentice Hall, Englewood Cliffs, NJ, Eq. (2-5.24).

- Hoffmann, M., Schlüter, M., Räbiger, N., 2006. Experimental investigation of liquid-liquid mixing in T-shaped micro-mixers using $\mu$-LIF and $\mu$-PIV. Chem. Eng. Sci. 61, 2968-2976.

- Hussong, J., Lindken, R., Pourquie, M., Westerweel, J., 2009. Numerical study on the flow physics of a T-shaped micro-mixer, IUTAM bookseries 15, 191-205.

- Jeong, J., Hussain, F., 1995. On the identification of a vortex. J. Fluid Mech. 285, 69-94.

- Kockmann N., Roberge, D.M., 2011. Transitional flow and related transport phenomena in curved microchannels, Heat Transfer Eng. 32, 595-608.

- Kumar, V., Paraschivoiu, M., Nigam, K.D.P., 2011. Single-phase fluid flow and mixing in microchannels, Chem. Eng. Sci. 66, 1329-1373.

- Laliberté, M., 2007. Model for calculating the viscosity of aqueous solutions, J. Chem. Eng. Data 52, 321-335.

- Lei, Q., Hou, Y.-C., 1999. Correlation of viscosity of binary liquid mixtures, Fluid Phase Equil. 154, 153 - 163.

- Middleman, S., 1998. An Introduction to Mass and Heat Transfer, Wiley, New York, p. 450. 
- Minakov, A.V., Rudyak, V.Y., Gavrilov, A.A., Dekterev, A.A., 2012. Mixing in a T-shaped micromixer at moderate Reynolds numbers. Thermophys Aeromech. 19, 385-395.

- Minakov, A.V., Rudyak, V.Y., Dekterev, A.A., Gavrilov, A.A., 2013. Investigation of slip boundary conditions in the T-shaped microchannel, Int. J. Heat Fluid Flow 43, 161-169.

- Orsi, G., Roudgar, M., Brunazzi, E., Galletti, C., Mauri, R., 2013. Waterethanol mixing in T-shaped microdevices, Chem. Eng. Sci. 95, 174-183.

- Orsi, G., Galletti, C., Brunazzi, E., Mauri, R., 2013. Mixing of two miscible liquids in T-shaped microdevices, Chem. Eng. Trans. 32, 1471-1476.

- Orsi, G., Mauri, R., 2013. Volume of mixing effect on fluid counterdiffusion, Phys. Fluids 25, 082101.

- Ottino, J.M., 1994. Mixing and chemical reactions a tutorial, Chem. Eng. Sci. 49, 4005 - 4027.

- Ottino, J.M., Chella, R., 1983. Laminar mixing of polymeric liquids; a brief review and recent theoretical developments, Polym. Eng. \& Science $23,357-379$.

- Simmonds, C., 1919. Alcohol, Its Production, Properties, Chemistry, and Industrial Applications, Macmillan, NewYork.

- Thomas, S., Ameel, T., Guilkey, J., 2010. Mixing kinematics of moderate Reynolds number flows in a T-channel, Phys. Fluids 22, 013601.

- Thomas, S., Ameel, T., 2010. An experimental investigation of moderate Reynolds number flow in a T-Channel, Exp. Fluids 49, 12311245. 


\section{List of Figures}

1 Viscosity as a function of composition for all investigated mixtures. 23

2 Sketch of the T-mixer. . . . . . . . . . . . . . . . 24

3 Vortical structures, as identified by the $\lambda_{2}$ criterion, and y-vorticity contours in the mixing channel cross-sections $y=1.5 d$ and $y=3 d$ for N1-type mixture at $R e=400$, obtained using different CFD codes: (a,c) Fluent v. 15; (b,d) Nek5000. . . . . . . . . . . . 25

4 Concentration field of a passive tracer in the cross-section $y=$ $1.5 d$ of the mixing channel, for different types of mixtures and Reynolds numbers. . . . . . . . . . . . . . 26

5 Vortical structures, as identified by the $\lambda_{2}$ criterion, and y-vorticity contours in the cross-sections $y=1.5 d$ and $y=3 d$ of the mixing channels, for different types of mixtures and Reynolds numbers. . 27

6 Vortical structures, as identified by the $\lambda_{2}$ criterion, and y-vorticity contours in the mixing channel cross-sections $y=1.5 d$ and $y=3 d$ at different times $t / \tau$, for N1-type mixtures at $R e=600$ and for N2-type mixtures at $R e=500 \ldots \ldots \ldots \ldots 28$

$7 \quad$ Vortical structures, as identified by the $\lambda_{2}$ criterion, and y-vorticity contours in the mixing channel cross-sections $y=1.5 d$ and $y=3 d$ at different times $t / \tau$, for P1-type mixtures at $R e=250$ and

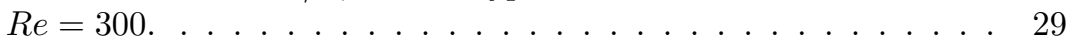

8 Time series of the $v_{y}$ velocity component at $y=0$ in the axis of the mixing channel for N1-type mixture and different Reynolds numbers: (a) $R e=550$; (b) $R e=600$; (c) $R e=650$; (d) $R e=700.30$

9 Strouhal number as a function of the Reynolds number. . . . . . 31

10 Degree of mixing as a function of the Reynolds number. Solid lines refer to steady (vortex and engulfment) flow regimes, while dotted lines with error bars refer to unsteady (time-periodic and chaotic) regimes. . . . . . . . . . . . . . . . 32 


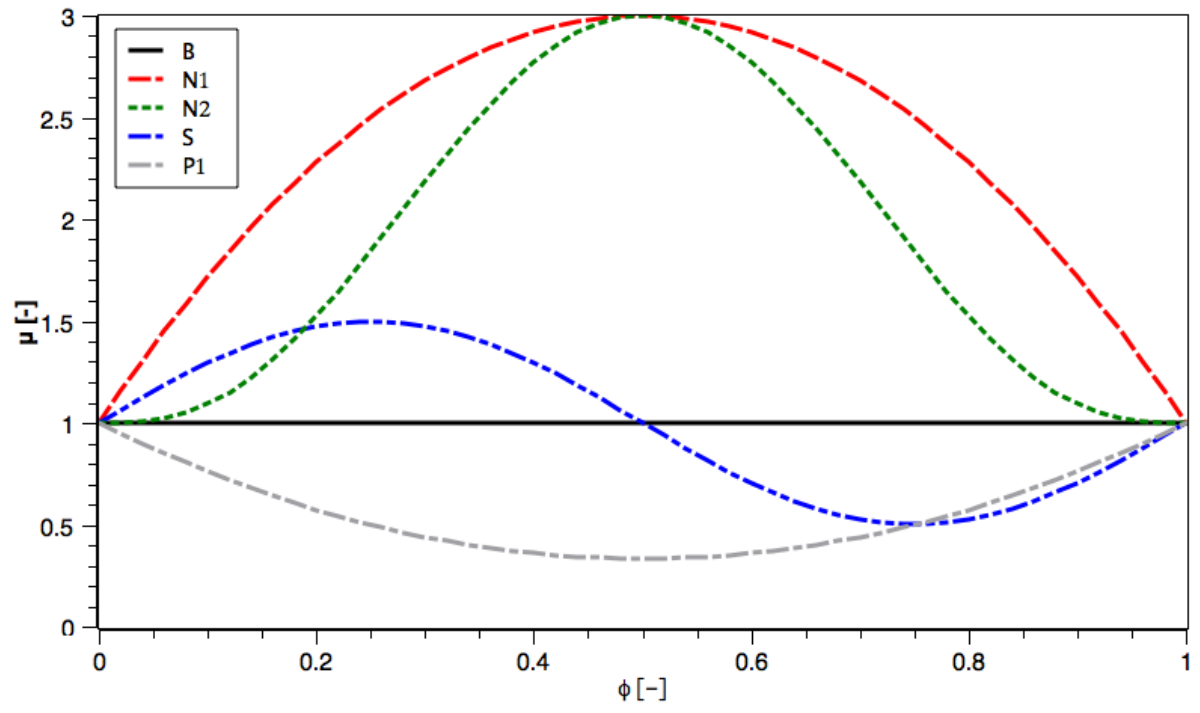

Figure 1: Viscosity as a function of composition for all investigated mixtures. 


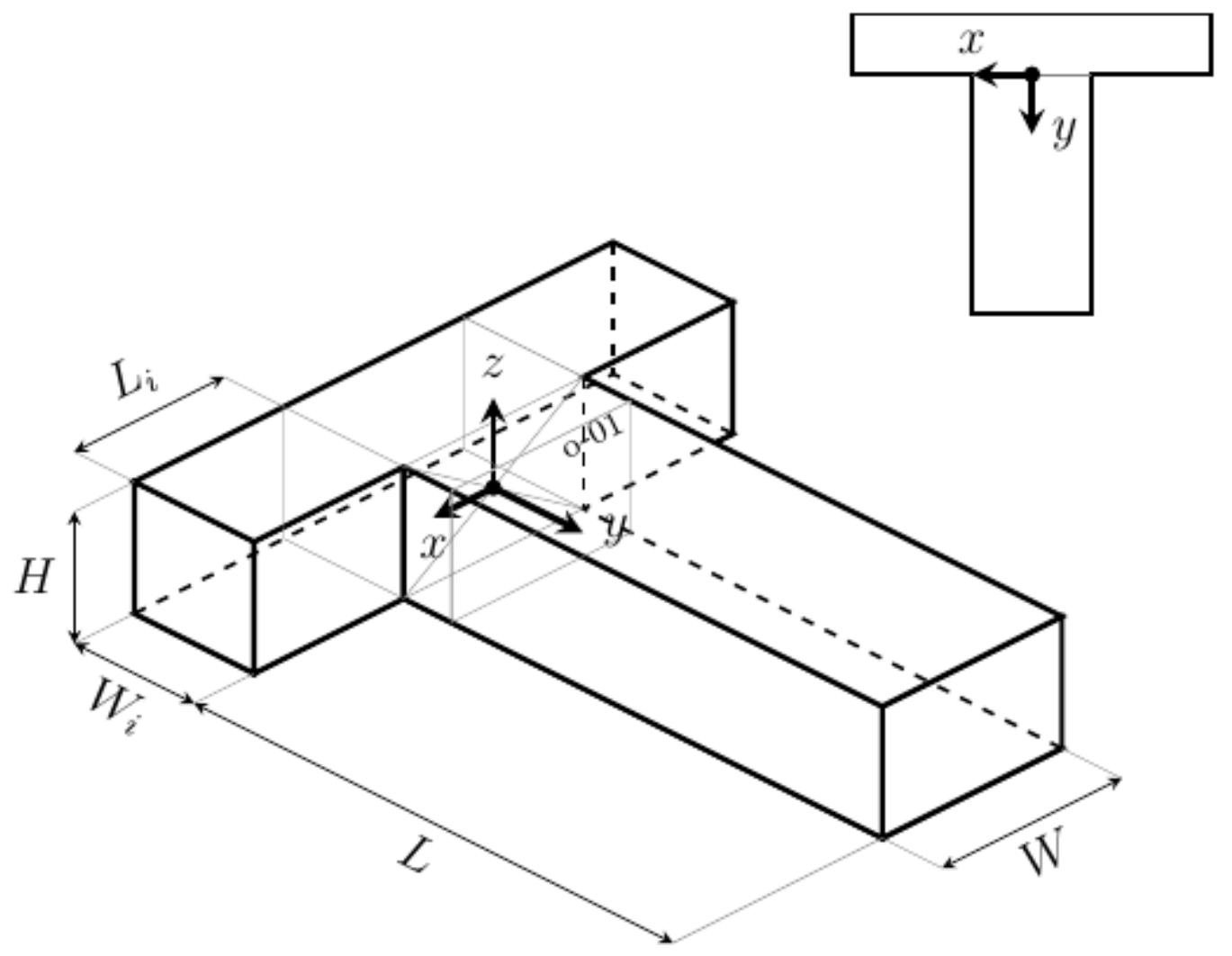

Figure 2: Sketch of the T-mixer. 
(a)

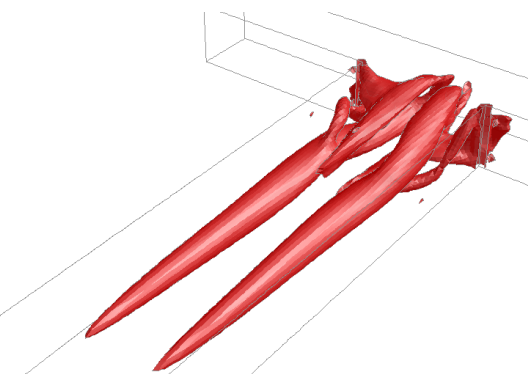

(c)

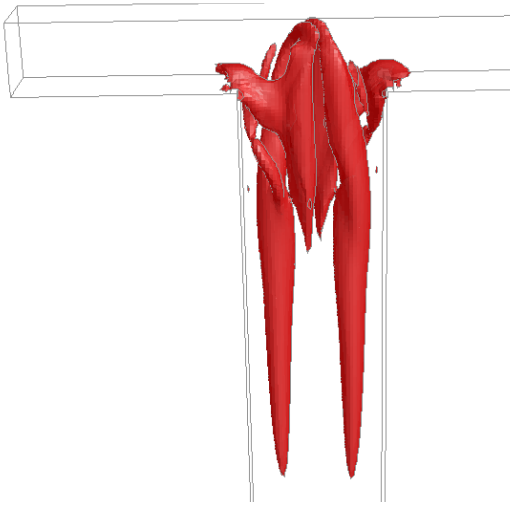

(b)

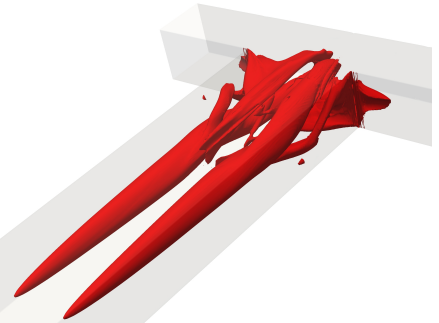

(d)

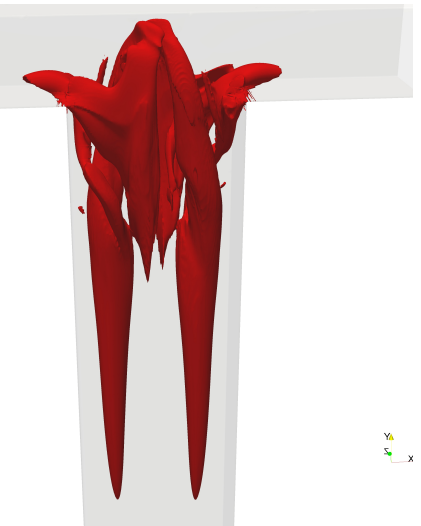

Figure 3: Vortical structures, as identified by the $\lambda_{2}$ criterion, and y-vorticity contours in the mixing channel cross-sections $y=1.5 d$ and $y=3 d$ for N1-type mixture at $R e=400$, obtained using different CFD codes: (a,c) Fluent v. 15; (b,d) Nek5000. 


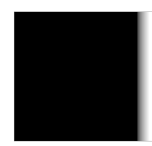

(a) $\mathrm{B}, \mathrm{Re}=125$

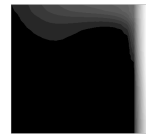

(e) $\mathrm{N} 1, \mathrm{Re}=125$

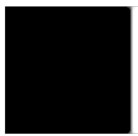

(i) $\mathrm{N} 2, \mathrm{Re}=125$

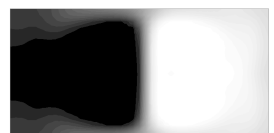

(m) S, Re $=125$

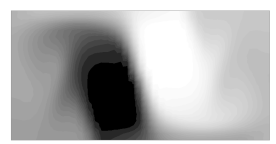

(q) $\mathrm{P} 1, \mathrm{Re}=125$

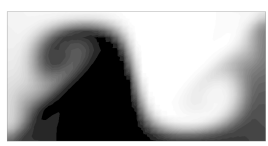

(b) $\mathrm{B}, \mathrm{Re}=150$

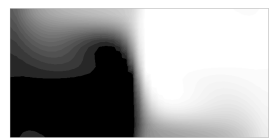

(f) $\mathrm{N} 1, \mathrm{Re}=150$

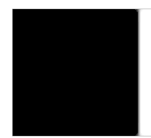

(j) $\mathrm{N} 2, \mathrm{Re}=150$

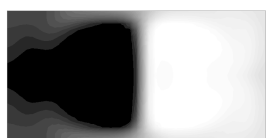

(n) $\mathrm{S}, \mathrm{Re}=150$

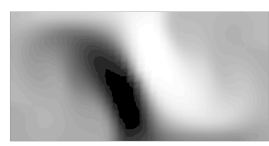

(r) $\mathrm{P} 1, \mathrm{Re}=150$

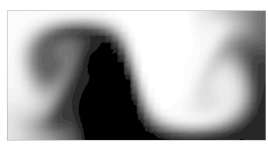

(c) $\mathrm{B}, \mathrm{Re}=160$

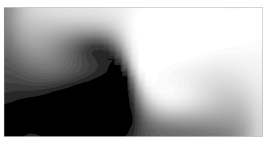

(g) N1, Re=160

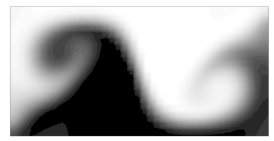

(k) $\mathrm{N} 2, \mathrm{Re}=160$

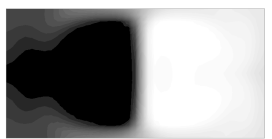

(o) $\mathrm{S}, \mathrm{Re}=160$

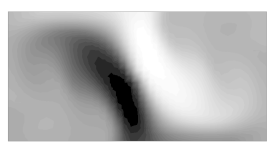

(s) $\mathrm{P} 1, \mathrm{Re}=160$

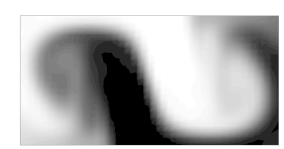

(d) $\mathrm{B}, \mathrm{Re}=175$

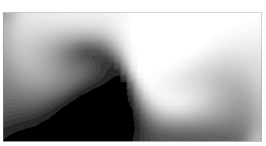

(h) $\mathrm{N} 1, \mathrm{Re}=175$

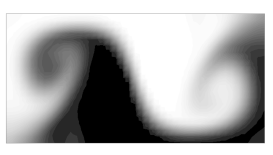

(l) $\mathrm{N} 2, \mathrm{Re}=175$

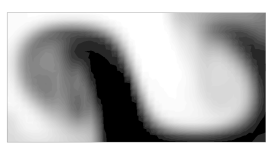

(p) $\mathrm{S}, \mathrm{Re}=175$

Figure 4: Concentration field of a passive tracer in the cross-section $y=1.5 d$ of the mixing channel, for different types of mixtures and Reynolds numbers. 


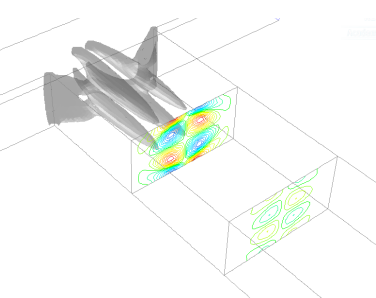

(a) $\mathrm{B}, \mathrm{Re}=125$

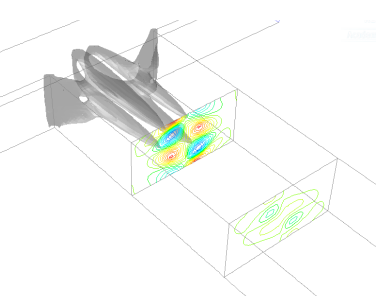

(d) N1, Re $=150$

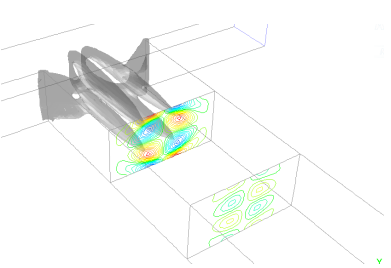

(g) N2, Re $=150$

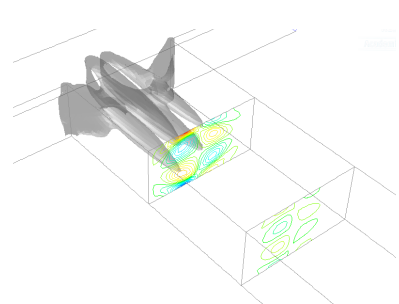

(j) $\mathrm{S}, \mathrm{Re}=125$

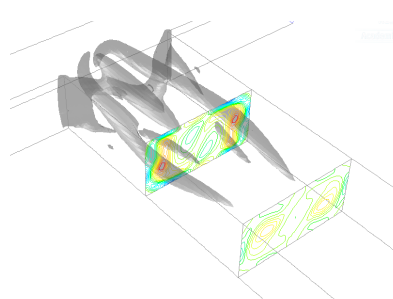

(m) P1, Re $=125$

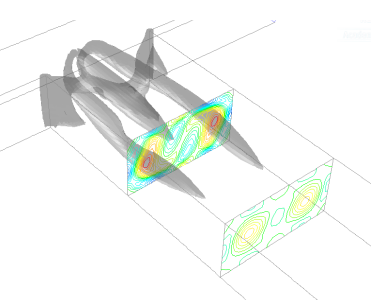

(b) $\mathrm{B}, \mathrm{Re}=175$

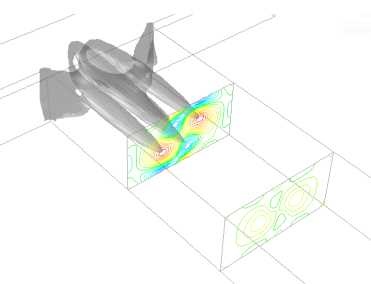

(e) N1, Re $=175$

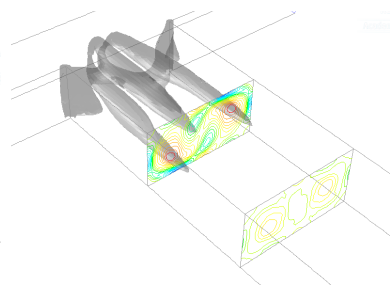

(h) N2, Re $=175$

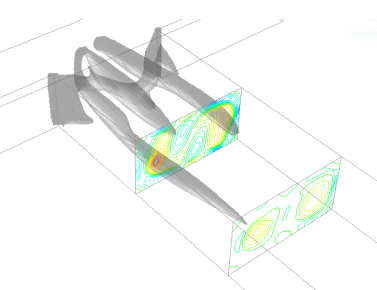

(k) S, Re $=167$

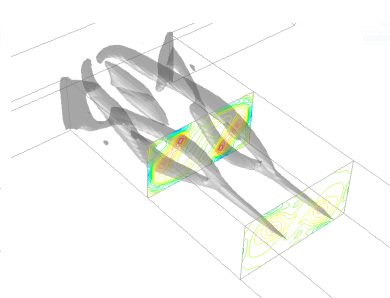

(n) $\mathrm{P} 1, \operatorname{Re}=150$

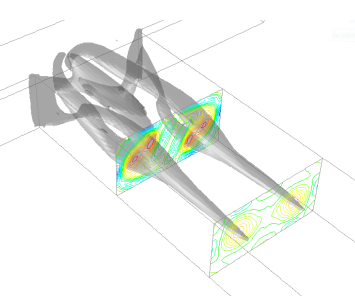

(c) $\mathrm{B}, \mathrm{Re}=225$

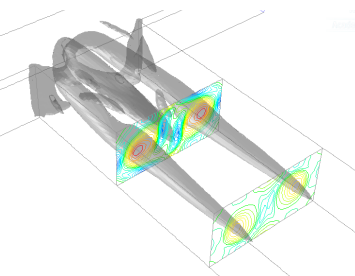

(f) $\mathrm{N} 1, \mathrm{Re}=400$

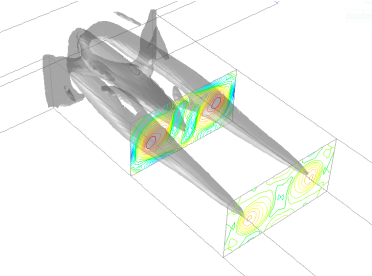

(i) $\mathrm{N} 2, \mathrm{Re}=300$

Figure 5: Vortical structures, as identified by the $\lambda_{2}$ criterion, and y-vorticity contours in the cross-sections $y=1.5 d$ and $y=3 d$ of the mixing channels, for different types of mixtures and Reynolds numbers. 


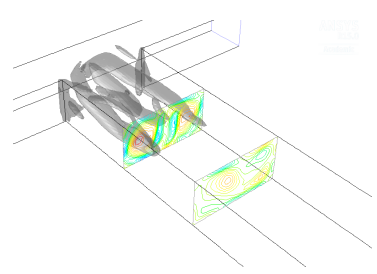

(a) $\mathrm{N} 1, t / \tau=0$

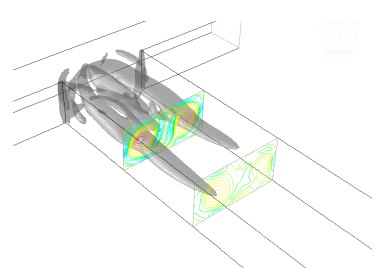

(c) $\mathrm{N} 1, t / \tau=0.2$

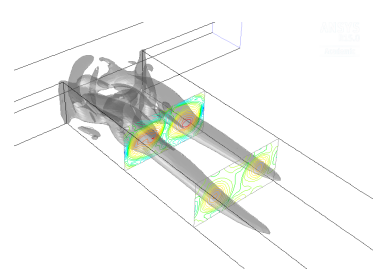

(e) $\mathrm{N} 1, t / \tau=0.4$

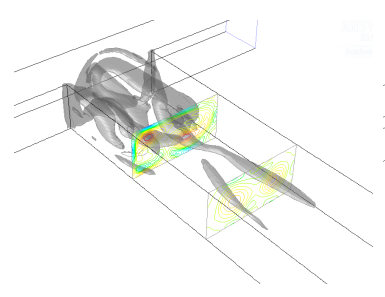

(g) $\mathrm{N} 1, t / \tau=0.6$

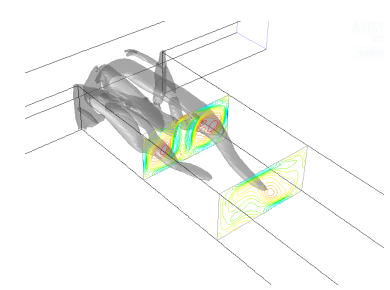

(i) $\mathrm{N} 1, t / \tau=0.8$

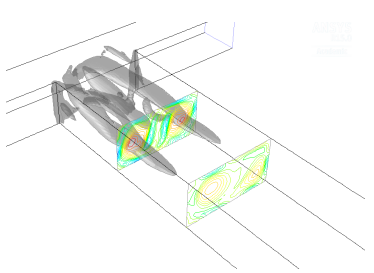

(b) $\mathrm{N} 2, t / \tau=0$

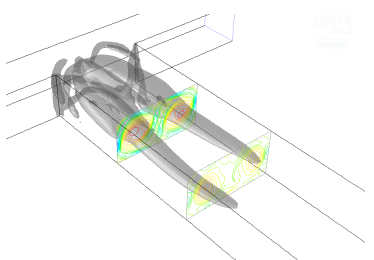

(d) $\mathrm{N} 2, t / \tau=0.2$

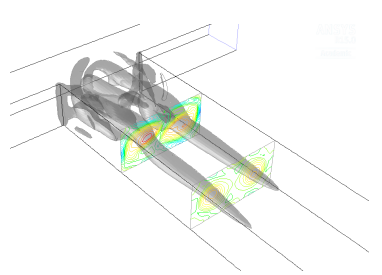

(f) $\mathrm{N} 2, t / \tau=0.4$

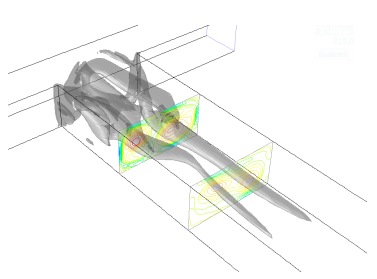

(h) $\mathrm{N} 2, t / \tau=0.6$

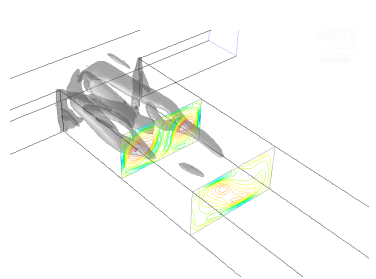

(j) $\mathrm{N} 2, t / \tau=0.8$

Figure 6: Vortical structures, as identified by the $\lambda_{2}$ criterion, and y-vorticity contours in the mixing channel cross-sections $y=1.5 d$ and $y=3 d$ at different times $t / \tau$, for N1-type mixtures at $R e=600$ and for N2-type mixtures at $R e=500$. 


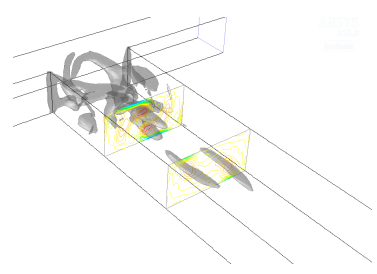

(a) $\mathrm{P} 1, \mathrm{Re}=250, t / \tau=0$

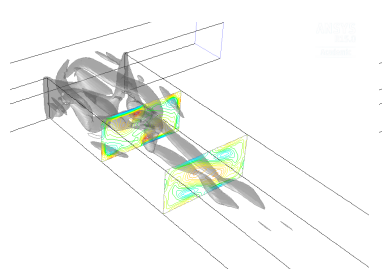

(c) $\mathrm{P} 1, \operatorname{Re}=250, t / \tau=0.2$

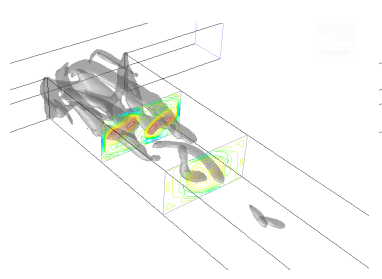

(e) $\mathrm{P} 1, \operatorname{Re}=250, t / \tau=0.4$

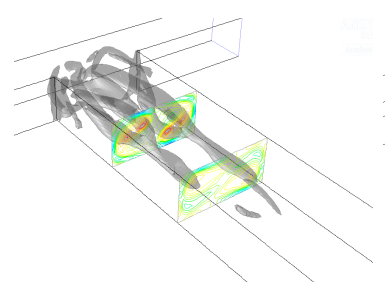

(g) $\mathrm{P} 1, \mathrm{Re}=250, t / \tau=0.6$

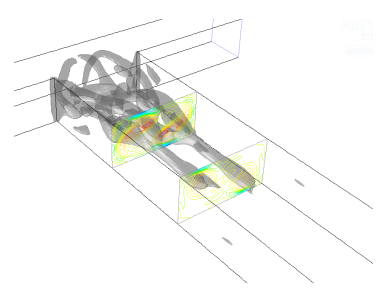

(i) $\mathrm{P} 1, \mathrm{Re}=250, t / \tau=0.8$

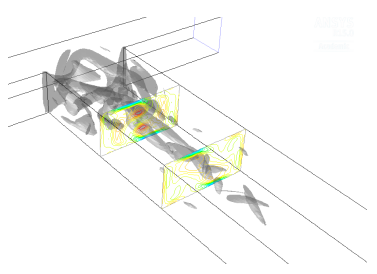

(b) P1, Re $=300, t / \tau=0$

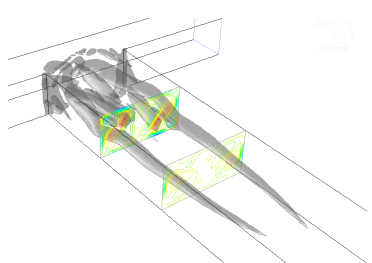

(d) $\mathrm{P} 1, \mathrm{Re}=300, t / \tau=0.2$

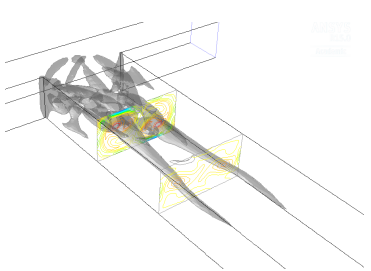

(f) $\mathrm{P} 1, \mathrm{Re}=300, t / \tau=0.4$

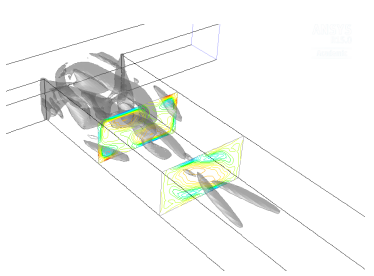

(h) $\mathrm{P} 1, \mathrm{Re}=300, t / \tau=0.6$

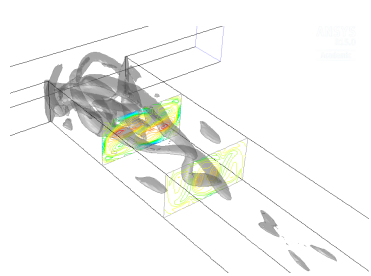

(j) P1, Re $=300, t / \tau=0.8$

Figure 7: Vortical structures, as identified by the $\lambda_{2}$ criterion, and y-vorticity contours in the mixing channel cross-sections $y=1.5 d$ and $y=3 d$ at different times $t / \tau$, for P1-type mixtures at $R e=250$ and $R e=300$. 


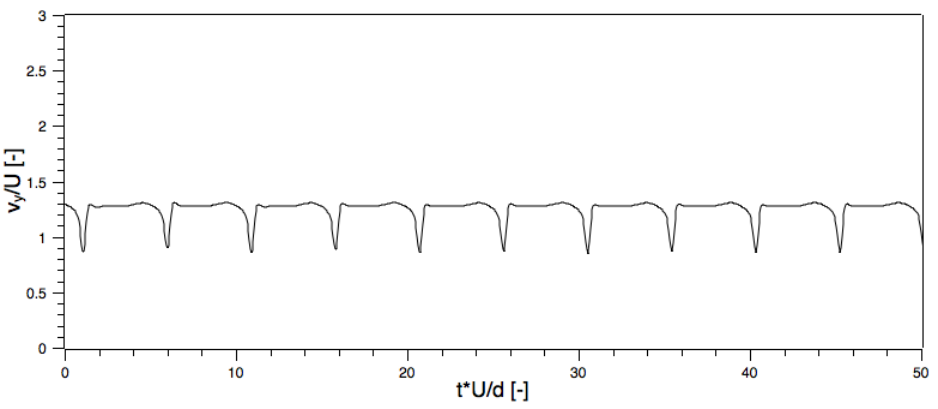

(a)

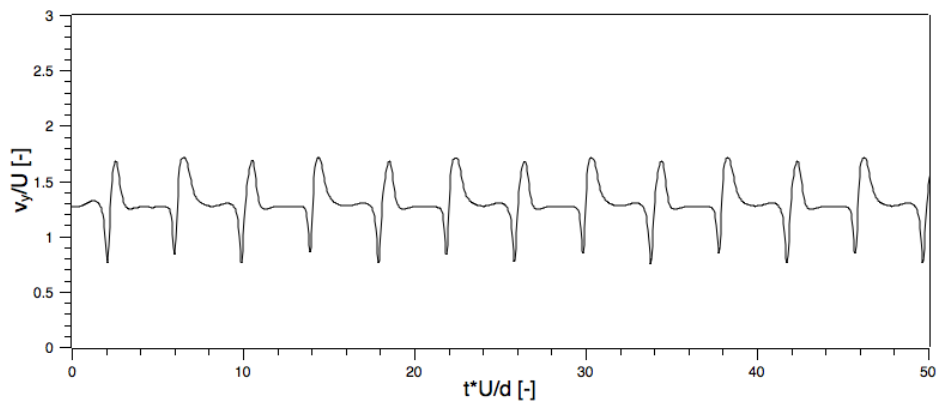

(b)

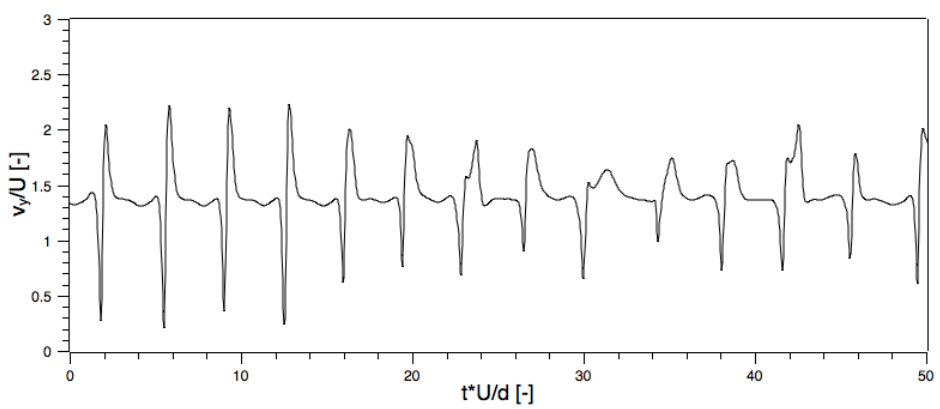

(c)

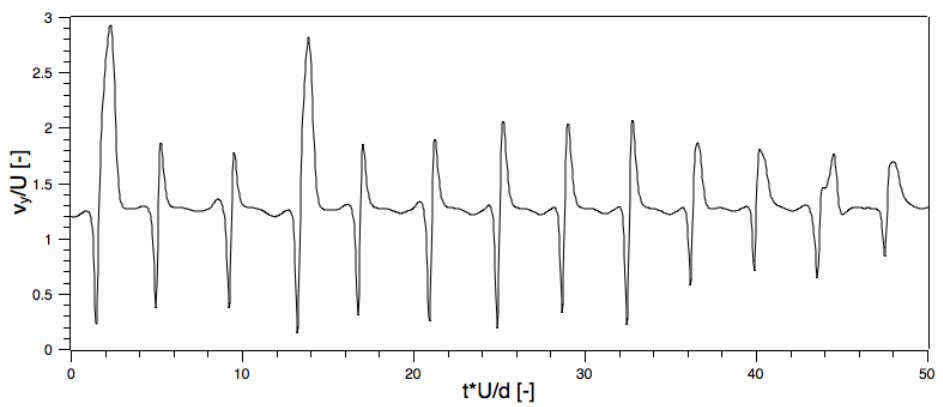

(d)

Figure 8: Time series of the $v_{y}$ velocity component at $y=0$ in the axis of the mixing channel for N1-type mixture and different Reynolds numbers: (a) $R e=550$; (b) $R e=600$; (c) $R e=650 ;$ (d) $R e=700$. 


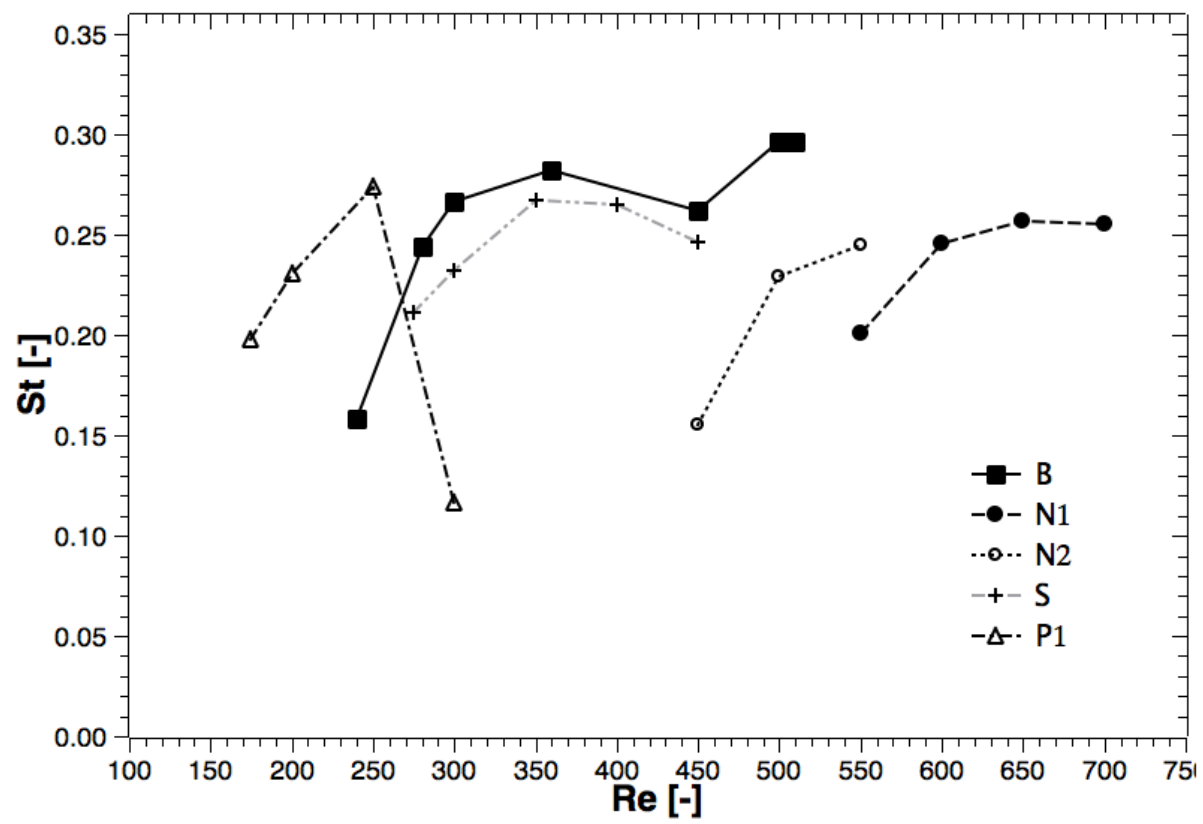

Figure 9: Strouhal number as a function of the Reynolds number. 


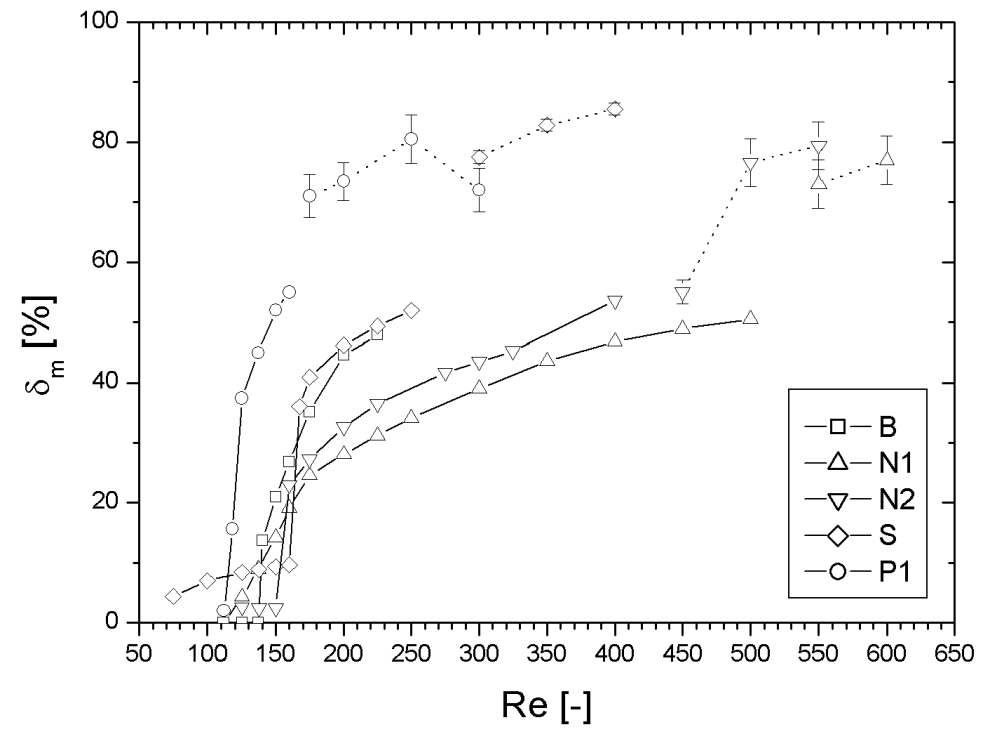

Figure 10: Degree of mixing as a function of the Reynolds number. Solid lines refer to steady (vortex and engulfment) flow regimes, while dotted lines with error bars refer to unsteady (time-periodic and chaotic) regimes. 


\section{List of Tables}

1 Reynolds numbers corresponding to the onset of the engulfment $\left(R e_{E}\right)$, time-periodic $\left(R e_{U}\right)$ and chaotic $\left(R e_{C}\right)$ regimes for the different types of mixture. . . . . . . . . . . . . 34 
Table 1: Reynolds numbers corresponding to the onset of the engulfment $\left(R e_{E}\right)$, time-periodic $\left(R e_{U}\right)$ and chaotic $\left(R e_{C}\right)$ regimes for the different types of mixture.

\begin{tabular}{c|ccc} 
mixture & $R e_{E}$ & $R e_{U}$ & $R e_{C}$ \\
\hline B0 & 138 & 240 & 515 \\
N1 & - & 550 & 650 \\
N2 & 150 & 450 & 550 \\
S & 167 & 300 & 450 \\
P1 & 118 & 175 & 350
\end{tabular}

